\title{
The prevalence of Adverse Drug Events in septic patients after Rapid Response Team Activation
}

SAAD AL QAHTANI ( $\square$ MCMASTERER@HOTMAIL.COM )

Research article

Keywords: RRT, antimicrobials, adverse events, antimicrobial stewardship, Saudi Arabia

Posted Date: January 24th, 2020

DOI: https://doi.org/10.21203/rs.2.21797/v1

License: (c) (1) This work is licensed under a Creative Commons Attribution 4.0 International License. Read Full License 


\section{Abstract}

Background: Antibiotics are commonly prescribed in hospitals, some use are unnecessary or inappropriate, which may increase Adverse Drug Events (ADEs) rate. ADEs can be a leading cause of morbidity and mortality, despite this; it is receiving little attention. A meta-analysis done by Lazarou. $J$ and colleagues, estimated that more than 2 million (6.7\%) of hospitalized patients have a serious adverse drug reaction (ADR) with a mortality rate of $0.32 \%$, means ADR causing over 100,000 deaths annually. This study was conducted to evaluate the prevalence of antibiotics ADEs in Critical Care Response Team (CCRT) activated patients.

Methods Single-center, randomized, prospective observational cohort study took place at an academic tertiary hospital in Riyadh, Saudi Arabia. All adult patients who received antibiotics were included in the study. ADE was defined as any recognized / possible injury associated with antimicrobials use and were evaluated post CCRT (RRT) activation. Results A total of 315 patients with a mean age of $61.1 \pm 20.4$ who received antibiotics mainly for pneumonia included in the study. $29.5 \%$ experienced at least one ADE through the study period. The most frequently common ADE were renal in $12.4 \%$, followed by the hematological adverse events in $11.4 \%$ of the patients.

Conclusion We found that antibiotics - associated ADEs were common in CCRT patients, minimizing the unnecessary use of antibiotics, following hospital antibiotic guideline and applying other necessary measures can decrease ADEs morbidity rate.

\section{Introduction}

CCRT is a multidisciplinary team consists of physician, nurse and respiratory therapist that bring high care service within an average of 15 minutes to the deteriorated patient on bedside in non-intensive care wards, evaluating and assessing patient's condition, making recommendations and interventions, and if required transferring the patient to the ICU, which saves hospital about $\$ 3500$ for each CCRT call. Some recommendations include adding and modifying medications which carries the risk of ADE. Risk factors associated with ADE in CCRT patients include admission to ICUs, older age, renal and hepatic impairment, length of hospital stay, polypharmacy and patient movement among wards. ${ }^{1,2,3}$

$1,2,3$

ADEs are a noticeable concern that can increase morbidity and mortality rate associated with medications use. ${ }^{24}$ They can lead to increase length of hospital stay, intensive care units (ICUs) admissions, ordering additional medications, and labs, which in turn increase healthcare costs. ${ }^{1,9} \mathrm{~A}$ study from Saudi Arabia showed an ADE incidence rate of $16 \%$ in four hospitals, about one third of these incidence events were preventable, of the preventable events, $11.9 \%$ were life-threatening and $1.2 \%$ was fatal. ${ }^{2}$ They reported a total 245 ADE cases, of which 51 cases related to antibiotics use. ${ }^{2}$ Antibiotics accounted as one of the most common medication classes associated with ADEs during hospital stay. Per JADE study, the overall mortality of antibiotics related ADE was ten out of 14 patients. ${ }^{3}$

Antibiotics are a commonly prescribed in hospitals, up to $50 \%$ of all prescribed antibiotics in US acute care hospitals are inappropriate per the Centers for Disease Control and Prevention (CDC). ${ }^{26-27}$ In 2007 to improve antimicrobial therapy and to optimize the use of antibiotics, the Infectious Disease Society of America (IDSA) and the Society for Healthcare Epidemiology of America (SHEA) published guidelines for developing an antimicrobial stewardship programs (AMS) program. ${ }^{28}$ AMS regulations have been updated and approved in January, 2017 by the Joint Commission. ${ }^{25}$ Clostridium difficile infection and resistant rate are indicators for AMS outcomes. ${ }^{25}$ In our hospital, the resistant rate is dramatically increasing, which may indicates inappropriate use of antibiotics. The U.S. malpractice lawsuits in 2016, mostly showed claims related to antibiotics. ${ }^{25}$ For this and because the needing of well-established ADE studies, we are aiming to examine the CCRT-ordered antimicrobial ADEs prevalence and to develop a plan to minimize those events.

\section{Methods}

Setting. This study took place at one of the largest hospitals in the area, King Abdulaziz Medical City (KAMC), an academic tertiary hospital with a bed capacity of 1501 in Riyadh, Saudi Arabia. The study was approved from King Abdullah International Medical Research Centre (KAIMRC) Institutional Review Board, with a waiver of informed consent from patients as there was no intervention and no more than minimal risk involved.

Population. All patients 15 years and older admitted to KAMC from FEB 2018 to JAN 2019, with CCRT activation who received antibiotics. Exclusion criteria included antimicrobials agents other than antibiotics or antifungal (e.g. antiviral), antibiotics with an indication other than infection (e.g. erythromycin for gastroparesis), and routs other than oral and intravenous.

Study design and Data collection. Single-center, prospective observational cohort study design used. Data were collected using a monitoring form, following characteristics were assessed: demographic data (table.1), date of CCRT activation, hospital admission/discharge dates, vital signs, 
antibiotics used, microbial culture, ADE (anaphylaxis, skin, hematological, renal, liver and others). CCRT team send a list of the patients have been visited in the prior week, CCRT coordinator will randomly include patients in the study. An infectious disease specialist physician and a clinical pharmacist meet weekly to determine the appropriateness of antibiotic use and the ADE incidence.

\section{Outcome definition}

Appropriateness of antimicrobial use: The use of an agent/s following MNGHA antimicrobial guidelines which maximizes clinical therapeutic effect while minimizing drug-related toxicity and the development antimicrobial resistance ${ }^{13}$

ADEs: any recognized injury/ potential injury associated with antimicrobials use and were evaluated post CCRT activation.

Acute kidney injury: Per KDIGO an increase in SCr by $\geq 0.3 \mathrm{mg} / \mathrm{dl}$ ( $\geq 26.5 \mu \mathrm{mol} / \mathrm{I})$ within 48 hours; or increase in SCr to $\geq 1.5$ times baseline, which is presumed to have occurred within the prior 7 days; or urine volume $\otimes 0.5 \mathrm{ml} / \mathrm{kg} / \mathrm{h}$ for at least 6 hours $^{14}$

Hepatic impairment: marked elevation in aminotransferase levels more than 10 times the upper reference limit, or moderate elevation with 5-10 times the upper reference limit ${ }^{15}$

Hematological ADE: platelet count drop by $50 \%$ from the baseline or below a count of $150,000 \mu$.

\section{Statistical methods}

Descriptive statistics were utilized to analyze the data. Continuous variables were summarized using mean \pm standard deviation

\section{Patients}

A total of 315 CCRT patients received antibiotics treatment were included in this cohort study. The most common indication for antibiotics was pneumonia. The mean age of the patients was $61.1 \pm 20.4$, and $54.6 \%$ participants were male. The most common admission diagnosis were cancer (52 [16.5\%]), renal related conditions (37 [11.7\%]), hepatobiliary and pneumonia of (34 [10.8\%]) and infection (32 [10.2\%]). The median length of hospital stay was 15 days (IQR, 10-30 days). The baseline characteristics are shown in Table 1.

Table 1. The baseline characteristics. 


\begin{tabular}{|c|c|}
\hline Characteristics & Mean (\%) \\
\hline Age & $61.1 \pm 20.4$ \\
\hline \multicolumn{2}{|l|}{ Gender: } \\
\hline Male & $172(54.6 \%)$ \\
\hline Female & $143(45.4 \%)$ \\
\hline \multicolumn{2}{|l|}{ BMI } \\
\hline$<30 \mathrm{~kg} / \mathrm{m}^{2}$ & $210(66.7 \%)$ \\
\hline$\geq 30 \mathrm{~kg} / \mathrm{m}^{2}$ & $105(33.3 \%)$ \\
\hline \multicolumn{2}{|l|}{ Service } \\
\hline Medical & $177(56.2 \%)$ \\
\hline Surgical & $68(21.6 \%)$ \\
\hline Oncology & $44(14.0 \%)$ \\
\hline Others & $26(8.2 \%)$ \\
\hline \multicolumn{2}{|l|}{ Admission diagnosis } \\
\hline Cancer/tumor & $52(16.5 \%)$ \\
\hline Renal & $37(11.7 \%)$ \\
\hline Hepatobiliary & $34(10.8 \%)$ \\
\hline Pneumonia & $34(10.8 \%)$ \\
\hline Infection/sepsis & $32(10.2 \%)$ \\
\hline Trauma/orthopedic & $28(8.9 \%)$ \\
\hline Cardiac & $23(7.3 \%)$ \\
\hline Other lung disease & $18(5.7 \%)$ \\
\hline Surgical/post-surgical & $18(5.7 \%)$ \\
\hline Stroke & $17(5.4 \%)$ \\
\hline Others & $51(16.2 \%)$ \\
\hline Length of stay (days) & $15(10-30)$ \\
\hline
\end{tabular}

\section{Antibiotics Used}

Through this study, we evaluated the antibiotics use before, during and after CCRT activation. Broad spectrum beta lactams and vancomycin were the most commonly used antibiotics (Table2). At any phase during the study, meropenem was the most frequent used antibiotics with a rate of $51.4 \%$, followed by vancomycin $41.3 \%$, and Piperacillin and tazobactam $28.6 \%$.

Table 2. Antibiotics. 


\begin{tabular}{lllll} 
& Before CCRT & By CCRT team & After CCRT & At any phase \\
\hline Meropenem & $25 \%$ & $38 \%$ & $37 \%$ & $51.4 \%$ \\
\hline Vancomycin & $14 \%$ & $30 \%$ & $20 \%$ & $41.3 \%$ \\
\hline Piperacillin and tazobactam & $18 \%$ & $15 \%$ & $16 \%$ & $28.6 \%$ \\
\hline Ceftriaxone & $10 \%$ & $4 \%$ & $2 \%$ & $12.1 \%$ \\
\hline Linezolid & $3 \%$ & $5 \%$ & $6 \%$ & $8.6 \%$ \\
\hline Colistin & $4 \%$ & $4 \%$ & $4 \%$ & $7.3 \%$ \\
\hline Caspofungin & $1 \%$ & $3 \%$ & $4 \%$ & $6.7 \%$ \\
\hline Anidulafungin & $2 \%$ & $2 \%$ & $4 \%$ & $5.7 \%$ \\
\hline Tigecycline & $3 \%$ & $3 \%$ & $3 \%$ & $5.4 \%$ \\
\hline Ciprofloxacin & $4 \%$ & $3 \%$ & $3 \%$ & $5.4 \%$ \\
\hline Imipenem & $3 \%$ & $3 \%$ & $2 \%$ & $4.4 \%$ \\
\hline Others & $41 \%$ & $37 \%$ & $28 \%$ & $70.5 \%$ \\
\hline
\end{tabular}

\section{Adverse Drug Event}

Total of 93 patients (29.5\%) experienced at least one adverse event. The most common organ affected by antibiotics used was the renal 39 (12.4\%), followed by the hematological adverse events in 36 patients (11.4\%), gastrointestinal tract in 24 (7.6\%), and hepatic with 14 (4.4\%) (Table.3).

Table 3.

\begin{tabular}{ll}
\multicolumn{2}{l}{ Total } \\
\hline Antimicrobial AE & \\
\hline None & $222(70.5 \%)$ \\
\hline One or more & $93(29.5 \%)$ \\
One & $72(22.9 \%)$ \\
Two or more & $21(6.7 \%)$ \\
\hline Antimicrobial AE & \\
\hline Renal & $39(12.4 \%)$ \\
\hline Hematologic & $36(11.4 \%)$ \\
\hline GIT & $24(7.6 \%)$ \\
\hline Hepatic & $14(4.4 \%)$ \\
\hline Skin & $4(1.3 \%)$ \\
\hline Anaphylaxis & $1(0.3 \%)$
\end{tabular}


Table 4. Other Outcomes.

\begin{tabular}{ll} 
Outcome & $\begin{array}{l}\text { Total patients } \\
\text { (315) }\end{array}$ \\
\hline Stay at floor & $171(54.3 \%)$ \\
\hline Admission to ICU & $114(36.2 \%)$ \\
\hline Mortality & $9(2.9 \%)$ \\
\hline Discharge Home & $2(0.6 \%)$ \\
\hline Others & $2(0.6 \%)$
\end{tabular}

\section{Discussion}

World Health Organization (WHO) identified ADE as "Medical occurrence temporally associated with the use of a medicinal product, but not necessarily causally related", unlike the Adverse Drug Reaction (ADR) which has a causal relationship with pharmaceutical product use that may requires discontinuing or changing therapy. ${ }^{1,4,5}$ U.S. Food and Drug Administration (FDA) defined ADE as "any undesirable experience associated with the use of a medical product in a patient". ${ }^{11}$ In a prospective cohort study, Bates and colleagues described ADE as a medical intervention injuries caused by a drug. ${ }^{12}$ For this study, the ADE was defined as any recognized or possible injury associated with antimicrobials use from the day of CCRT activation.

ADEs can occur because of multiple medication use, in particular the use of four or more medications. ${ }^{33} \mathrm{Jacubeit}^{\mathrm{T}}$ and colleagues found a positive correlation between the ADRs incidence and the number of prescription per patient, with no effect of age. ${ }^{32}$ Drug interaction can occur in several mechanism, is another factor contribute for up to $5 \%$ of the total ADEs. ${ }^{33}$ Here in Ministry of National Guard - Health Affairs (MNGHA), we use systemic reporting system (SRS) for reporting ADEs. In 2006, medication safety program was launched to improve patient safety.

Antimicrobial Stewardship Program was established to ensure proper antimicrobial use. In a national level, SFDA (Saudi FDA) encourages health care professionals to report ADRs through SFDA-National Pharmacovigilance and Drug Safety Center reporting system. In one month, only one ADR was reported from our institution to the SFDA reporting system which indicates a poor reporting practice, and it is expected to have a greater number.

The next crucial step of this study is developing strategies to implement ADEs prevention criteria. Proposed ways to reduce ADEs rates and improve safety may include, emphasizing the importance of using appropriate antimicrobial agent and avoid unnecessary usage to healthcare providers by ensuring to achieve the "4 D's of optimal antimicrobial therapy" suggested by Joseph J and Rodvold KA that include; right Drug, Dose, De-escalation to pathogen, and Duration of therapy ${ }^{31}$. Utilizing information technology, Bates ${ }^{19}$ suggested computerized adverse drug event detection, as self-reporting was only $5 \%$ of ADEs. Prevent the excessive and unnecessary alert of drug interactions, to minimize the high overridden practice that may happen to an important and high priority drug interaction. ${ }^{33}$ Actively involved clinical pharmacist in rounds showed to reduce medications errors, duplicates therapies, unnecessary ordering and optimize drug therapy plan $1,17,18,20$. In addition, the involvement of a trained pharmacist in the rapid response team / CCRT has shown to improve medication accessibility, help optimize medication recommendation, dosing, medication related causes of deterioration within 15 minutes for each call. ${ }^{29,} 30$ Implement effective medication reconciliation process which is recognized by Joint Commission, January 2018 as an essential goal for patient safety. ${ }^{21,22}$ Encourage collaboration between ASP and Medication Safety Program to assess the insinuation ADEs as an outcome measurement to achieve their mission. 


\section{Abbreviations}

ADR

Adverse Drug Reaction, $\mathrm{ADE}=$ Adverse Drug Event, $\mathrm{CCRT}=$ Critical Care Response Team, RRT $=$ Rapid Response Team

\section{Declarations}

Not applicable

Abbreviations:

ADR= Adverse Drug Reaction, $A D E=$ Adverse Drug Event, $C C R T=$ Critical Care Response Team, RRT= Rapid Response Team

Ethics approval and consent to participate:

Not applicable

Consent for publication:

Yes

Availability of data and material:

The datasets used and/or analysed during the current study are available from the corresponding author on reasonable request.

Competing interests:

None

Funding :

None

Authors' contributions:

SA contributed to the study design and writing. SA and KA contributed to the study design and critical review. KA contributed to data collection and analysis. Both authors read and approved the final manuscript.

Acknowledgements:

we thank Prof. Hanan H. Balkhy and Dr. Aiman El-Saed for there help in reviewing the manuscript.

\section{References}

1. Adverse Drug Reactions By Stephanie N. Schatz, Pharm.D., BCPS; and Robert J. Weber, Pharm.D., BCPS

2. https://www.accp.com/docs/bookstore/psap/2015B2.SampleChapter.pdf

3. Incidence of adverse drug events in public and private hospitals in Riyadh, Saudi Arabia: the (ADESA) prospective cohort study. Hisham Aljadhey

4. https://www.ncbi.nlm.nih.gov/pmc/articles/PMC4947792/

5. Incidence of Adverse Drug Events and Medication Errors in Japan: the JADE Study. Takeshi Morimoto, MD,

6. https://www.ncbi.nlm.nih.gov/pmc/articles/PMC3019321/

7. WHO Definitions (cited 2014). http://www.who.int/medicines/areas/quality_safety/safety_efficacy/trainingcourses/definitions.pdf 
8. American Society of Health-System Pharmacists. ASHP guidelines on adverse drug reaction monitoring and reporting. Am J Health Syst Pharm 1995;52:417-9.

9. Ann Pharmacotherapy. 2008 Jul;42(7):1017-25. doi: 10.1345/aph.1L037. Epub 2008 Jul 1. Hospital admissions associated with adverse drug reactions: a systematic review of prospective observational studies.

10. https://www.ncbi.nIm.nih.gov/pubmed?term=2008\%5Bpdat\%5D+AND+Kongkaew+C\%5Bfirst+author\%5D\&cmd=detailssearch

11. https://sfda.gov.sa/ar/drug/about/sector_departments/national_pharmacovigilance_center/Documents/SFDApharmacovigilancesystem.pdf

12. Characteristics of Adverse Drug Events Originating During the Hospital Stay, 2011

13. https://www.hcup-us.ahrq.gov/reports/statbriefs/sb164.jsp

14. Ann Intern Med. 2004 May 18;140(10):795-801. Clarifying adverse drug events: a clinician's guide to terminology, documentation, and reporting. Nebeker JR1, Barach P, Samore MH. https://www.ncbi.nlm.nih.gov/pubmed?

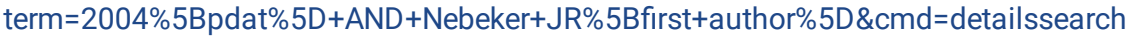

15. https://www.fda.gov/Safety/MedWatch/HowToReport/ucm053087.htm

16. Bates, David W., et al. "Incidence of adverse drug events and potential adverse drug events: implications for prevention." Jama1 (1995): $29-34$.

17. World Health Organization. (2001). WHO global strategy for containment of antimicrobial resistance.

18. http://www.kdigo.org/clinical_practice_guidelines/pdf/KDIGO\%20AKI\%20Guideline.pdf

19. Giannini, E. G., Testa, R., \& Savarino, V. (2005). Liver enzyme alteration: a guide for clinicians. Canadian medical association journal, 172(3), 367-379.

20. Sekhon, S. S., \& Roy, V. (2006). Thrombocytopenia in adults: a practical approach to evaluation and management. Southern medical journal, 99(5), 491-499.

21. https://www.wisconsinmedicalsociety.org/_WMS/publications/wmj/pdf/114/2/83.pdf

22. Am J Health Syst Pharm. 2002 Nov 1;59(21):2089-92. Pharmacist participation in medical rounds reduces medication errors.

23. Using information technology to reduce rates of medication errors in hospitals David W Bates BMJ

24. 1999 Jul 21;282(3):267-70. Pharmacist participation on physician rounds and adverse drug events in the intensive care unit. Leape LL1, Cullen DJ, Clapp MD, Burdick E, Demonaco HJ, Erickson JI, Bates DW.

25. J Hosp Med. 2010 Oct;5(8):477-85. doi: 10.1002/jhm.849. Making inpatient medication reconciliation patient centered, clinically relevant and implementable: a consensus statement on key principles and necessary first steps. Greenwald JL1, Halasyamani L, Greene J, LaCivita C,

Stucky E, Benjamin B, Reid W, Griffin FA, Vaida AJ, Williams MV. January 2018,

https://www.jointcommission.org/assets/1/6/NPSG_Chapter_AHC_Jan2018.pdf

26. https://ade.sfda.gov.sa/FAQs.aspx

27. Classen, D. C. (1997). Adverse drug events in hospitalized patients. Excess length of stay, extra costs, and attributable mortality. JAMA, 277(4), 301-306. doi:10.1001/jama.277.4.301

28. https://www.jointcommissionjournal.com/article/S1553-7250(17)30545-7/fulltext

29. https://www.jointcommission.org/assets/1/6/New_Antimicrobial_Stewardship_Standard.pdf

30. https://www.cdc.gov/antibiotic-use/healthcare/implementation/core-elements.html

31. https://www.pfizer.com/files/news/Antibiotic_Stewardship_Final_Oct_2016.pdf

32. J Pharm Pract. 2016 Apr;29(2):116-20. doi: 10.1177/0897190014544794. Epub 2014 Aug 8. Pharmacists as Members of the Rapid Response Team. Groth CM1, Acquisto NM2.

33. Am J Health Syst Pharm. 2017 Mar 1;74(5 Supplement 1):S10-S16. doi: 10.2146/ajhp160076. Pharmacist involvement on a rapid response team. Feih J1, Peppard WJ2, Katz M3. 